\title{
Bundles on non-proper schemes: representability
}

\section{Vladimir Baranovsky}

(C) The Author(s) 2010. This article is published with open access at Springerlink.com

\begin{abstract}
Let $X$ be a proper scheme over a field $k$ which satisfies Serre's condition $S_{2}$ and $G$ a reductive group over $k$. We prove that the functor of principal $G$-bundles, defined away from a non-fixed closed subset in $X$ of codimension at least 3 , is an algebraic stack in the sense of Artin.
\end{abstract}

Keywords Moduli of vector bundles $\cdot$ Principal bundles $\cdot$ Artin stacks

Mathematics Subject Classification (2000) Primary 14D20; Secondary 14D23

\section{Introduction}

Let $X_{k}$ be a proper irreducible scheme over a field $k$ which satisfies Serre's $S_{2}$ condition (for the sake of simplicity the reader may think that $X_{k}$ is smooth or a locally complete intersection). For a noetherian scheme $T$ over $k$ denote $X_{T}=\left(X_{k}\right) \times \operatorname{Spec}(k) T$ and let $\Phi(T)$ be the collection of all closed subsets $Z \subset X_{T}$ such that every point $z \in Z$ has codimension $\geq 3$ in its fiber over $T$. Fix a reductive group $G$ over $k$.

Definition 1 In the notation above, let $F_{G}(T)$ be a groupoid category with the objects ( $E, U)$ where $E$ is a principal $G$-bundle defined on an open subscheme $U \subset X_{T}$,

\footnotetext{
The main result of this paper was conjectured by Vladimir Drinfeld whom the author thanks for useful discussions and encouragement. Thanks are also due to Victor Ginzburg for helping to clarify the role of Serre's $S_{2}$ condition (which is closely related to the concept of a reflexive sheaf), and to the reviewer for pointing out several sloppy statements and gaps in the proofs of the original version of this paper. This work was supported by the Sloan Research Fellowship and a UCI Teaching Relief Grant.
}

\footnotetext{
V. Baranovsky ( $\square)$

Department of Mathematics, UC Irvine, Irvine, CA 92697, USA

e-mail: vbaranov@math.uci.edu
} 
such that the closed complement of $U$ is a subset in $\Phi(T)$. A morphism $\left(E_{1}, U_{1}\right) \rightarrow$ $\left(E_{2}, U_{2}\right)$ is an isomorphism $\left.\left.E_{1}\right|_{W} \simeq E_{2}\right|_{W}$ on an open subset $W \subset U_{1} \cap U_{2}$ such that the complement of $W$ is again in $\Phi(T)$. The composition of morphisms is defined in an obvious way.

For any morphism $\alpha: T^{\prime} \rightarrow T$ of schemes over $T$ we have pullback functors $\alpha^{*}: F_{G}(T) \rightarrow F_{G}\left(T^{\prime}\right)$ satisfying the usual compatibility conditions for any pair of morphisms $T^{\prime \prime} \stackrel{\beta}{\longrightarrow} T^{\prime} \stackrel{\alpha}{\longrightarrow} T$, i.e., $F_{G}$ is a groupoid over the category of noetherian schemes over $k$, cf. Sect. 1 in [1]. As usual, we will mostly deal with its restriction to affine noetherian schemes over $k$, writing $X_{A}$ and $\Phi(A)$ instead of $X_{\operatorname{Spec}(A)}$ and $\Phi(\operatorname{Spec}(A))$, respectively. The main goal of this paper is the following result.

Theorem $2 F_{G}$ is an algebraic stack, locally of finite type over $k$, such that the diagonal morphism $\Delta: F_{G} \rightarrow F_{G} \times \operatorname{Spec}(k) F(G)$ is separated and quasi-compact.

Thus we obtain a partial compactification of the stack of $G$-bundles on $X_{k}$. Our strategy of proof is straightforward, if seldom used: we apply Artin's representability criterion, cf. Theorem 5.3 in [1] for a statement and [2,3,13] for examples of application.

For the most part of the paper (see Sects. 3-7) we consider the case of vector bundles, i.e., work with $G=G L(r)$ for fixed $r \geq 1$; and write $F$ instead of $F_{G}$. In Sect. 8 we show how the proof is extended to the case of general $G$ and also explain why the result fails when the "codimension 3" condition in the definition of $\Phi(T)$ is replaced by "codimension 2 ".

\section{Depth and local cohomology}

We will freely use definitions and basic properties of local cohomology and depth that can be found, e.g., in Sect. 18 and Appendix 4 to [6] and Sects. I-III of [15]. Observe that the scheme $X_{T}$ will not satisfy Serre's $S_{2}$ condition since no depth assumptions are made for $T$. However, we can formulate a relative version of this condition.

Definition 3 For any point $x \in X_{T}$ let $d(x)$ be the codimension of $x$ in its fiber over $T$.

Lemma 4 Let $E$ be a vector bundle on an open subset $U \subset X_{T}$ and $M$ a coherent sheaf on $T$. For $t \in T$ let $X_{t}$ be the fiber of $X_{T}$ over $t$ and suppose that for every $t$ the structure sheaf of the intersection $U_{t}=U \cap X_{t}$ satisfies Serre's condition $S_{n}$. Set $E_{M}=E \otimes_{\mathcal{O}_{T}} M$. Then for any $x \in U$ one has $\operatorname{depth}_{U, x} E_{M} \geq \min (n, d(x))$.

Definition 5 We will call the inequality stated in this lemma the relative $S_{n}$ condition. In this paper $n=2$ or 3 .

Proof First we recall the following result, cf. Proposition 1.2.16 in [5]: suppose $\phi$ : $(R, \mathfrak{m}) \rightarrow(S, \mathfrak{n})$ is a local homomorphism of local noetherian rings, $M$ is a finitely generated $R$-module, and $N$ is a finitely generated $S$-module which is flat over $R$. Then

$$
\operatorname{depth}_{S}\left(M \otimes_{R} N\right)=\operatorname{depth}_{R}(M)+\operatorname{depth}_{S}(N / \mathfrak{m} N) .
$$


Now let $x \in U$ be a point, $t \in T$ its image, and take $R=\mathcal{O}_{T, t}, S=\mathcal{O}_{U, x}, N=E_{x}$. By the result quoted, $\operatorname{depth}_{U, x} E_{M} \geq \operatorname{depth}_{U \cap X_{t}, x}\left(\left.E\right|_{U \cap X_{t}}\right) \geq \min (n, d(x))$ where the second inequality is due to the $S_{n}$ assumption of the lemma and the fact that $E$ is locally free.

Below, dealing with obstructions, deformations, and infinitesimal automorphisms, we need the following construction: For any coherent sheaf $E$ on $X_{T}$ set

$$
H_{T, \Phi}^{i}(E)=\underline{\lim }_{\{Z \in \Phi(T)\}} H^{i}\left(X_{T} \backslash Z, E\right)
$$

where the filtered direct limit is taken with respect to the inclusion of closed subsets $Z \subset Z^{\prime}$ in $\Phi(T)$. If $T=\operatorname{Spec}(A)$ is affine we write $H_{A, \Phi}^{i}(E)$ instead of $H_{\operatorname{Spec}(A), \Phi}^{i}(E)$. If $(E, U)$ is a pair representing an object in $F(T)$, we set

$$
H_{T, \Phi}^{i}(E, U)=\underline{\lim }_{\left\{Z \in \Phi(T) \mid Z_{0} \subset Z\right\}} H^{i}\left(X_{T} \backslash Z, E\right)
$$

where $Z_{0}=X_{T} \backslash U \in \Phi(T)$. It is clear that if $\left(E_{1}, U_{1}\right),\left(E_{2}, U_{2}\right)$ are two objects of $F(T)$, then an isomorphism of $\left.E_{1}\right|_{U_{1} \cap U_{2}}$ and $\left.E_{2}\right|_{U_{1} \cap U_{2}}$ induces an isomorphism $H_{T, \Phi}^{i}\left(E_{1}, U_{1}\right) \simeq H_{T, \Phi}^{i}\left(E_{2}, U_{2}\right)$. In particular, shrinking $U$ does not change these groups (as long as the complement of $U$ is in $\Phi(T)$ ), and we will abuse notation by writing $H_{T, \Phi}^{i}(E)$ instead of $H_{T, \Phi}^{i}(E, U)$. We will only use these definitions for $i=0,1,2$. The next result shows that under certain conditions the cohomology groups stabilize for $i=0,1$, and for $i=2$, the limit eventually reduces to an increasing union.

Lemma 6 With the notation just introduced, assume that $Z \subset Z^{\prime}$ are in $\Phi(T)$. If $E$ satisfies the relative $S_{2}$ condition on $X_{T} \backslash Z$, then the natural restriction morphism

$$
\rho_{i}: H^{i}\left(X_{T} \backslash Z, E\right) \rightarrow H^{i}\left(X_{T} \backslash Z^{\prime}, E\right)
$$

is an isomorphism for $i=0$ and injective for $i=1$. If, in addition, E satisfies the relative $S_{3}$ condition on $X_{T} \backslash Z$, then $\rho_{i}$ is an isomorphism for $i=0,1$ and injective for $i=2$. Moreover, there exists a closed $Z_{T}^{\circ} \in \Phi(T)$ such that the relative $S_{3}$ condition on $X_{T} \backslash Z$ does hold when $Z_{T}^{\circ} \subset Z$.

Proof Denote $U=X_{T} \backslash Z, W=U \cap Z^{\prime}$ and consider the spectral sequence of local cohomology $H^{p}\left(U, \mathcal{H}_{W}^{q}(E)\right) \Rightarrow H_{W}^{p+q}(U, E)$. By the relative $S_{2}$ condition the local cohomology sheaves $\mathcal{H}_{W}^{i}(E)$ vanish for $i=0$, 1 , while the relative $S_{3}$ condition at the points of $W$ also implies $\mathcal{H}_{W}^{2}(E)=0$. Now the assertion follows from the standard long exact sequence

$$
\cdots \rightarrow H_{W}^{i}(U, E) \rightarrow H^{i}(U, E) \rightarrow H^{i}(U \backslash W, E) \rightarrow H_{W}^{i+1}(U, E) \rightarrow \cdots
$$

Observe that the relative $S_{3}$ condition on $E_{M}$ also holds if $Z$ contains

$$
Z_{T}^{\circ}=Z^{\circ} \times \operatorname{Spec}(k) T \in \Phi(T)
$$


where $Z^{\circ} \subset X_{k}$ is the set of all points in $X_{k}$ where the $S_{3}$ condition fails for the structure sheaf. Observe that $Z^{\circ}$ is closed by [9], Proposition 6.11.2; since its complement contains all points of codimension $\leq 2$ we indeed have $Z_{T}^{\circ} \in \Phi(T)$.

Corollary 7 If $Z_{T}^{\circ} \subset Z$, then

$$
H_{T, \Phi}^{i}\left(E_{M}\right)=H^{i}\left(X_{T} \backslash Z, E_{M}\right)
$$

for $i=0,1$ and $E_{M}=E \otimes_{T} M$ as above. Moreover, if $j$ stands for the open embed$\operatorname{ding} X_{T} \backslash Z \hookrightarrow X_{T}$, then the sheaves $j_{*} E_{M}$ and $R^{1} j_{*} E_{M}$ are coherent on $X_{T}$ and if $T=\operatorname{Spec}(A)$ is affine the two stable cohomology groups are finitely generated A-modules.

Proof Stabilization follows immediately from the previous lemma. Coherence of the two direct images is due to [15], VII.2.3 while the finite generation is proved by combining the spectral sequence $H^{p}\left(X_{T}, R^{q} j_{*}\left(E_{M}\right)\right) \Rightarrow H^{p+q}\left(X_{T} \backslash Z, E_{M}\right)$ with the fact that $X_{T}$ is proper over $T$.

Remark Of course, for $i=2$ even the cohomology group $H^{2}\left(\mathbb{P}_{k}^{3} \backslash P, \mathcal{O}\right)$ is infinite dimensional over $k$, for any closed point $P$.

\section{Locally finite presentation}

In this section we do not use the $S_{2}$ assumption on $X_{k}$. Let $R=\lim _{\longrightarrow} R_{\alpha}$ be a filtered direct limit of $k$-algebras. We will assume that $R$ and $R_{\alpha}$ are noetherian, since this suffices in order to apply Artin's criterion in [1].

\section{Proposition 8}

$$
\lim _{\longrightarrow} F\left(R_{\alpha}\right) \rightarrow F(R)
$$

is an equivalence of categories.

Proof The assertion means that any object $(E, U) \in F(R)$ is isomorphic to the image of some $\left(E_{\alpha}, U_{\alpha}\right) \in F\left(R_{\alpha}\right)$ and that, whenever $\left(E_{\alpha}, U_{\alpha}\right)$ and $\left(E_{\beta}, U_{\beta}\right)$ give isomorphic objects in $F(R)$, there exists $\gamma$ such that $\gamma \geq \alpha, \gamma \geq \beta$ and the corresponding objects in $F\left(R_{\gamma}\right)$ are isomorphic. In addition, a similar condition should hold for morphisms: take a pair of objects $\left(E_{\alpha}, U_{\alpha}\right),\left(E_{\alpha}^{\prime}, U_{\alpha}^{\prime}\right)$ in $F\left(R_{\alpha}\right)$ and let $(E, U),\left(E^{\prime}, U^{\prime}\right)$ be the induced pair of objects in $F(R)$. Then for any isomorphism $\phi:\left.E\right|_{W} \simeq E_{W}^{\prime}$ over appropriate $W \subset U \cap U^{\prime}$ there exists $\beta \geq \alpha$, an open subset $W_{\beta} \subset U_{\beta} \cap U_{\beta}^{\prime}$ with closed complement in $\Phi\left(R_{\beta}\right)$, and an isomorphism $\phi_{\beta}:\left.\left.E_{\beta}\right|_{W_{\beta}} \simeq E_{\beta}^{\prime}\right|_{W_{\beta}}$ of the bundles $E_{\beta}, E_{\beta}^{\prime}$ induced by $E_{\alpha}, E_{\alpha}^{\prime}$, respectively, which induces the isomorphism $\phi$ after the base change. In addition, if for two indices $\beta, \gamma \geq \alpha$ the isomorphisms $\left(\phi_{\beta}, W_{\beta}\right)$, $\left(\phi_{\gamma}, W_{\gamma}\right)$ induce the same isomorphism $(\phi, W)$ in $F(R)$, then there exists an index $\delta$ such that $\delta \geq \beta, \delta \geq \gamma$ and the images of $\left(\phi_{\beta}, W_{\beta}\right),\left(\phi_{\gamma}, W_{\gamma}\right)$ in $F\left(R_{\delta}\right)$ are equal.

To prove the assertion for objects, consider a vector bundle $E$ on $U \subset X_{R}$ and take a finite affine covering $\left\{U_{i}\right\}$ of $U$ such that $\left.E\right|_{U_{i}}$ is trivial. 
Using the results of Sects. 8.2-8.5 of [10], we see that there exists $\alpha$ and open subsets $U_{i}^{\alpha}$ such that $U_{i}=\pi_{\alpha}^{-1}\left(U_{i}^{\alpha}\right)$ where $\pi_{\alpha}: X_{R} \rightarrow X_{R_{\alpha}}$ is the natural projection. Since in general a scheme $W$ is affine iff the canonical morphism $W \rightarrow \operatorname{Spec}\left(\Gamma\left(W, \mathcal{O}_{W}\right)\right)$ is an isomorphism, by increasing $\alpha$ if necessary we can assume that all $U_{i}^{\alpha}$ are affine.

The bundle $E$ is given by a cocycle $\left\{\phi_{i j} \in G L_{r}\left(U_{i} \cap U_{j}\right)\right\}_{i, j}$. Each $\phi_{i j}$ can be viewed as an automorphism of the trivial bundle; hence we can apply the usual approximation tools to it: increasing $\alpha$ we can assume that $\phi_{i j}$ come from $\phi_{i j}^{\alpha} \in G L_{r}\left(U_{i}^{\alpha} \cap U_{j}^{\alpha}\right)$. Increasing $\alpha$ again we can assume that $\phi_{i j}^{\alpha}$ satisfy the cocycle condition and thus define a vector bundle $E^{\alpha}$ on $U^{\alpha}=\bigcup_{i} U_{i}^{\alpha}$. By construction, $E \simeq \phi_{\alpha}^{*} E^{\alpha}$. To show that the closed complement $Z^{\alpha}$ of $U^{\alpha}$ is in $\Phi\left(\operatorname{Spec}\left(R_{\alpha}\right)\right.$ ) (again, after a possible increase of $\alpha$ ) note that $U=\pi_{\alpha}^{-1}\left(U^{\alpha}\right)$ and the fibers of $X_{R} \rightarrow \operatorname{Spec}(R)$ are obtained from the fibers $X_{R_{\alpha}} \rightarrow \operatorname{Spec}\left(R_{\alpha}\right)$ by extension of scalars. Therefore, the closed subset $W$ of points $s \in \operatorname{Spec}\left(R_{\alpha}\right)$ for which codimension of $Z^{\alpha} \cap X_{s}$ is $\leq 2$ has empty preimage in $\operatorname{Spec}(R)$. Therefore, for some $\alpha^{\prime} \geq \alpha$ the preimage of $W$ in $\operatorname{Spec}\left(R_{\alpha^{\prime}}\right)$ is empty and we can replace $\alpha$ by $\alpha^{\prime}$.

To prove surjectivity on morphisms, let $(E, U)$ and $\left(E^{\prime}, U^{\prime}\right)$ be two objects in $F(R)$, induced by a pair of objects $\left(E_{\alpha}, U_{\alpha}\right)$ and $\left(E_{\alpha}^{\prime}, U_{\alpha}^{\prime}\right)$ in $F\left(R_{\alpha}\right)$, and suppose we are given an isomorphism $\phi:\left.\left.E\right|_{W} \simeq E^{\prime}\right|_{W}$ where $W \subset U_{1} \cap U_{2}$ is open and its closed complement is in $\Phi(R)$. Since we assumed that $R$ is noetherian, $W$ is quasicompact. Hence, increasing $\alpha$ we can assume that $W$ is induced by the open subset $W_{\alpha} \subset U_{\alpha} \cap U_{\alpha}^{\prime}$. Then $\left.E_{\alpha}\right|_{W_{\alpha}}$ and $\left.E_{\alpha}^{\prime}\right|_{W_{\alpha}}$ become isomorphic after pullback to $U$; hence, by [10] increasing $\alpha$ we can find an isomorphism $\phi_{\alpha}:\left.\left.E_{\alpha}\right|_{U_{\alpha}} \simeq E_{\alpha}^{\prime}\right|_{U_{\alpha}}$ which induces $\phi$ on $U$. As before, we may have to increase $\alpha$ one more time to ensure that the complement of $U_{\alpha}$ is in $\Phi\left(R_{\alpha}\right)$.

Injectivity on morphisms is an immediate consequence of Theorem 8.5.2 in [10].

\section{Small affine pushouts}

Let $A_{0}$ be a noetherian $k$-algebra, and $A^{\prime} \rightarrow A$ a surjection of two infinitesimal extensions of $A_{0}$ such that $M=\operatorname{ker}\left(A^{\prime} \rightarrow A\right)$ is a finite $A_{0}$ module. Let $B$ be a noetherian ring and $B \rightarrow A$ a morphism, such that the composition $B \rightarrow A \rightarrow A_{0}$ is surjective.

Denote by $B^{\prime}$ the fiber product $A^{\prime} \times{ }_{A} B$, i.e., the subset of pairs $(a, b) \in A^{\prime} \times B$ which have the same image in $A$. Then $B^{\prime} \rightarrow B$ is surjective and its kernel may be identified with $M$ viewed as a $B$-module. Observe that the pushout $\operatorname{Spec}\left(B^{\prime}\right)$ is homeomorphic to $\operatorname{Spec}(B)$, while $\operatorname{Spec}\left(A^{\prime}\right), \operatorname{Spec}(A)$ and $\operatorname{Spec}\left(A_{0}\right)$ are homeomorphic to each other, and $\operatorname{Spec}\left(A_{0}\right) \rightarrow \operatorname{Spec}(B)$ is naturally a closed subscheme by the assumption.

Fix an object $a=\left(E_{A}, U_{A}\right) \in F(A)$. Let $F_{a}(B)$ be the groupoid of extensions of $a$ over $\operatorname{Spec}(B)$, and similarly for $A^{\prime}, B^{\prime}$.

Proposition 9 The natural functor

$$
F_{a}\left(B^{\prime}\right) \rightarrow F_{a}\left(A^{\prime}\right) \times F_{a}(B)
$$

is an equivalence of groupoids. 
Proof Suppose that $\left(E_{A^{\prime}}, U_{A^{\prime}}\right),\left(E_{B}, U_{B}\right)$ are two extensions of $(E, U)$ to $X_{A^{\prime}}$ and $X_{B}$, respectively. First show that these two extensions are induced by the same object $\left(E_{B^{\prime}}, U_{B^{\prime}}\right)$ in $F_{a}\left(B^{\prime}\right)$.

Since $\operatorname{Spec}(A)$ and $\operatorname{Spec}\left(A^{\prime}\right)$ are homeomorphic, shrinking $U_{A^{\prime}}, U_{B}$ and $U_{A}$, if necessary, we can assume $U_{A^{\prime}} \simeq U_{A} \simeq U_{B} \cap X_{A_{0}}$ (homeomorphisms induced by the natural embeddings). Observe that shrinking of the open sets does not change the isomorphism class of the objects in $F_{a}(-)$ by definition of the functor $F$. Denote by $U_{B^{\prime}}$ the subset $U_{B}$ viewed as an open subset of $X_{B^{\prime}}$. We have a commutative diagram

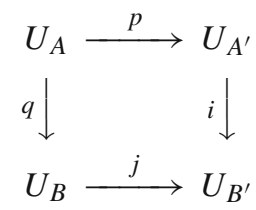

where the horizontal arrows are homeomorphisms. Since $q^{*} E_{B} \simeq E_{A}, p^{*} U_{A^{\prime}} \simeq E_{A}$ and $i p=j q$ there will be an exact sequence on $U_{B^{\prime}}$

$$
i_{*} E_{A^{\prime}} \oplus j_{*} E_{B} \rightarrow(i p)_{*} E_{A} \rightarrow 0
$$

where the first arrow is given by the difference of the obvious canonical maps. One can check that the kernel $E_{B^{\prime}}$ of the first arrow is a locally free sheaf of rank $r$ on $U_{B^{\prime}} \subset X_{B^{\prime}}$ such that $i^{*} E_{B^{\prime}} \simeq E_{A^{\prime}}, j^{*} E_{B^{\prime}} \simeq E_{B},(i p)^{*} E_{B^{\prime}} \simeq E_{A}$ in a compatible way.

Thus the functor $F_{a}\left(B^{\prime}\right) \rightarrow F_{a}\left(A^{\prime}\right) \times F_{a}(B)$ is essentially surjective on objects. A straightforward check shows that it is also bijective on morphisms, which finishes the proof.

\section{Automorphisms, deformations, obstructions}

We keep the notation of Sect. 4 , and recall that for a finitely generated $A_{0}$-module $M$ and a vector bundle $E$ we denote $E_{M}=E \otimes_{A_{0}} M$.

\subsection{Aut, D,O}

Infinitesimal automorphisms Let $A=A_{0}, A^{\prime}=A_{0} \oplus M$. Any $a_{0} \in F\left(A_{0}\right)$ given by a pair $(E, U)$ admits a trivial extension to $A_{0} \oplus M$ defined by $E^{\prime}=E \oplus E_{M}$. We are interested in the group of automorphisms Aut $_{a_{0}}\left(A_{0}+M\right)$ of the bundle $E^{\prime}$, which restrict to identity over $A_{0}$. Every such automorphism is defined uniquely by a morphism $E \rightarrow E_{M}$ defined, perhaps, on a smaller open subset $V \subset U$. In other words,

$$
\operatorname{Aut}_{a_{0}}\left(A_{0}+M\right)=H_{A_{0}, \Phi}^{0}\left(\operatorname{End}(E)_{M}\right)
$$

By Corollary 7 this is a finitely generated module over $A_{0}$. 
Deformations Now consider $D_{a_{0}}(M)$, the set of isomorphism classes of extensions of $a_{0}=(E, U)$ to $A^{\prime}$. If we fix the open subset $U \subset X_{A}$ and consider those extensions which are defined over $U$ by the standard results of deformation theory, cf. e.g., Proposition IV.3.1.5, isomorphism classes of such extensions are in bijective correspondence with $H^{1}\left(U, \operatorname{End}(E)_{M}\right)$. If $U^{\prime} \subset U$ is a smaller open subset such that $X_{A} \backslash U^{\prime}$ is in $\Phi(A)$, then the restriction of extended bundles from $U$ to $U^{\prime}$ agrees with the restriction morphism $H^{1}\left(U, \operatorname{End}(E)_{M}\right) \rightarrow H^{1}\left(U^{\prime}, \operatorname{End}(E)_{M}\right)$. By Lemma 6 there exists an open subset $U^{\circ}$ such that the restriction morphism on cohomology is an isomorphism if $U^{\circ} \supset U$, and in this case one has $H^{1}\left(U^{\circ}, \operatorname{End}(E)_{M}\right) \simeq H_{A_{0}, \Phi}^{1}\left(\operatorname{End}(E)_{M}\right)$. Thus, $D_{a_{0}}(M)=H_{A_{0}, \Phi}^{1}\left(\operatorname{End}(E)_{M}\right)$ which is also finitely generated over $A_{0}$, as established in Corollary 7.

Obstructions We are to define a finitely generated submodule of $O_{a}(M) \subset H_{A, \Phi}^{2}$ $\left(\operatorname{End}(E)_{M}\right)$ which will serve as obstruction module for our problem. Thus we consider a square zero extension $0 \rightarrow M \rightarrow A^{\prime} \rightarrow A \rightarrow 0$ and $a=\left(E_{A}, U_{A}\right) \in F(A)$. To define $O_{a}(M)$ first shrink $U_{A}$ so that its complement contains the closed subset $Z_{A}^{\circ}$ of Lemma 6. Then by the same lemma $H_{A_{0}, \Phi}^{2}\left(\operatorname{End}(E)_{M}\right)$ is an increasing union of $\operatorname{Ext}_{U^{\prime}}^{2}\left(\operatorname{End}\left(E_{A}\right), M\right)$ over the directed system of $U^{\prime}$ contained in $U_{A}$.

Next we recall, cf. Sect. IV.2.3.6 in [12], the Atiyah class of $E_{A}$

$$
a\left(E_{A}\right) \in \operatorname{Ext}_{U_{A}}^{1}\left(\operatorname{End}\left(E_{A}\right), L_{U_{A}}\right) .
$$

Here $L_{U_{A}}$ is the cotangent complex of $U$ over $k$, cf. Chapter II.1.2 of [12]. Since $X_{A}$ is a direct product of $X_{k}$ and $\operatorname{Spec}(A)$, its cotangent complex over $k$ splits into a direct sum $L_{X_{k}} \oplus L_{A}$ of the pullbacks of cotangent complexes from $X_{k}$ and $\operatorname{Spec}(A)$, respectively. Since $L_{X_{A}}$ restricts to $L_{U_{A}}$ we can define a composition of morphisms

$$
\operatorname{Ext}_{U_{A}}^{1}\left(L_{A}, M\right) \rightarrow \operatorname{Ext}_{U_{A}}^{1}\left(L_{U_{A}}, M\right) \rightarrow \operatorname{Ext}_{U_{A}}^{2}\left(\operatorname{End}\left(E_{A}\right), M\right)
$$

where the first arrow is induced by the direct sum splitting and the second by the Yoneda product with the Atiyah class. Denote by $O_{a}(M)$ the image of the above composition. Since the complement of $U_{A}$ contains $Z_{A}^{\circ}$, it can be viewed as a submodule of $H_{A_{0}, \Phi}^{2}\left(\operatorname{End}(E)_{M}\right)$. Observe that by Lemma 6 this submodule is independent on the choice of the open subset $U_{A}$ as long as $Z_{A}^{\circ}$ stays in its complement.

Lemma 10 The submodule $O_{a}(M)$ is finitely generated over $A_{0}$. For any square zero extension $A^{\prime}$ as above there is an obstruction class $\omega\left(E_{A}\right) \in \operatorname{Ext}_{U_{A}}^{2}\left(\operatorname{End}\left(E_{A}\right), M\right)$ which is zero if and only if $\left(E_{A}, U_{A}\right)$ admits a deformation to an object of $F\left(A^{\prime}\right)$. In this case the isomorphism classes of such deformations form a pseudo-torsor over $D_{a}(M)$.

Proof Since the cotangent complex of $\operatorname{Spec}(A)$ is concentrated in non-positive degrees and has finitely generated cohomology, we can find a quasi-isomorphic complex of free modules of finite rank $\cdots \rightarrow L_{2} \rightarrow L_{1} \rightarrow L_{0} \rightarrow 0$. The standard spectral sequence $E_{1}^{p, q}=\operatorname{Ext}_{U_{A}}^{p}\left(L_{q}, M\right) \Rightarrow \operatorname{Ext}_{U_{A}}^{p+q}\left(L_{A}, M\right)$ shows that $\operatorname{Ext}_{U_{A}}^{1}\left(L_{A}, M\right)$ has a two-step filtration with associated graded quotients depending only on $E_{1}^{p, q}$ for $p \leq 1$ and $q \leq 2$. Since the associated graded quotients of this filtration are subquotients of the terms with $p+q=1$, by Corollary $7, O_{a}(M)$ is finitely generated over $A$. 
Let $U_{A^{\prime}} \subset X_{A^{\prime}}$ be the open subset homeomorphic to $U_{A}$, with its natural structure of an open subscheme of $X_{A^{\prime}}$. By Proposition IV.3.1.8. of [I] the obstruction to deforming $E_{A}$ over $U_{A^{\prime}}$ is given by a class $\omega\left(E_{A}\right) \in \operatorname{Ext}_{U_{A}}^{2}\left(\operatorname{End}\left(E_{A}\right), M\right)$ which is a Yoneda product of two classes

$$
a\left(E_{A}\right) \in \operatorname{Ext}_{U_{A}}^{1}\left(\operatorname{End}\left(E_{A}\right), L_{U_{A}}\right), \quad \kappa\left(U / U^{\prime}\right) \in \operatorname{Ext}_{U_{A}}^{1}\left(L_{U_{A}}, M\right) .
$$

Here, $\kappa\left(U_{A} / U_{A^{\prime}}\right) \in \operatorname{Ext}_{U_{A}}^{1}\left(L_{U_{A}}, M\right)$ is the Kodaira-Spencer class of $U_{A^{\prime}}$ viewed as a deformation of $U_{A}$.

Since $X_{A^{\prime}}$ and $U_{A^{\prime}}$ viewed as deformations of $X_{A}$ and $U_{A}$, respectively, are induced by $\operatorname{Spec}(A) \rightarrow \operatorname{Spec}\left(A^{\prime}\right)$, the Kodaira-Spencer class $\kappa\left(U_{A} / U_{A^{\prime}}\right)$ belongs to the direct factor $\operatorname{Ext}_{U_{A}}^{1}\left(L_{A}, M\right) \subset \operatorname{Ext}_{U}^{1}\left(L_{U_{A}}, M\right)$. Therefore, $\omega\left(E_{A}\right)$ is the image of the Kodaira-Spencer class in $\operatorname{Ext}_{U_{A}}^{1}\left(L_{A}, M\right)$ under the map involved in the definition of $O_{a}(M)$ before this lemma.

Hence if the obstruction class vanishes, then $E_{A}$ can be deformed to a bundle over $U_{A}^{\prime}$ which, in particular, gives an object of $F\left(A^{\prime}\right)$. On the other hand, if a deforming object $\left(E^{\prime \prime}, U^{\prime \prime}\right)$ exits in $F\left(A^{\prime}\right)$, then shrinking $U^{\prime \prime}$ we can assume that $U^{\prime \prime} \subset$ $U_{A}^{\prime}$. By stabilization of Corollary 7 and the above spectral sequence converging to $\operatorname{Ext}_{U_{A}}^{p+q}\left(L_{A}, M\right)$ we see that the obstruction class vanishes, since all relevant cohomology groups are the same for $U^{\prime \prime}$ and $U_{A}^{\prime}$, and a deformation over $U^{\prime \prime}$ does exist. Repeating the argument in the proof of Proposition IV.3.1.2 in [I] (see page 248 of the same book) we also see that when the obstruction $\omega\left(E_{A}\right)$ vanishes, all deformations of $E_{A}$ over $U_{A^{\prime}}$ for a pseudo-torsor over $D_{a_{0}}(M)$, as claimed.

Assume now that $A$ is itself an extension

$$
0 \rightarrow N \rightarrow A \rightarrow A_{0} \rightarrow 0
$$

where $N$ is nilpotent and acts on $M$ by zero (so that $M$ is an $A_{0}$-module). Then the $A$-module structure on $O_{a}(M)$ descends to $A_{0}$ (i.e., $N$ acts trivially), hence $O_{a}(M)$ is a finitely generated $A_{0}$-module.

\subsection{Etale localization, completions, constructibility}

Let $p: A \rightarrow B$ be etale and consider $p_{0}: A_{0} \rightarrow B_{0}$ defined by $B_{0}=B \otimes_{A} A_{0}$, $p_{0}=p \otimes_{A} A_{0}$. Consider $a=\left(E_{A}, U_{A}\right) \in F(A)$ and let $b$ be its pullback in $F(B)$, and similarly for $a_{0}=(E, U) \in F\left(A_{0}\right), b_{0} \in F\left(B_{0}\right)$.

Proposition 11 There exist natural isomorphisms

$$
O_{b}\left(M \otimes_{A_{0}} B_{0}\right) \simeq O_{a}(M) \otimes_{A_{0}} B
$$

and

$$
\begin{aligned}
A u t_{b_{0}}\left(B_{0}+M \otimes_{A_{0}} B_{0}\right) & \simeq A u t_{a_{0}}\left(A_{0}+M\right) \otimes_{A_{0}} B_{0} \\
D_{a_{0}}\left(M \otimes_{A_{0}} B_{0}\right) & \simeq D_{a_{0}}(M) \otimes_{A_{0}} B_{0}
\end{aligned}
$$


Proof For Aut and $D$ we use their identification with $H^{i}\left(U, \operatorname{End}(E)_{M}\right)$ for $i=0,1$ and some $U \subset X_{A_{0}}$, see Corollary 7, and étale localization for cohomology. For $O$, let $\mathcal{Q}$ be a complex with finitely generated cohomology in non-positive degrees (defined up to quasi-isomorphism) which represents the class $a^{\prime}(E) \in \operatorname{Ext}_{U_{A}}^{1}\left(\operatorname{End}\left(E_{A}\right), L_{A}\right)$ obtained by projecting the Atiyah class $a(E)$ in $\operatorname{Ext}_{U_{A}}^{1}\left(\operatorname{End}\left(E_{A}\right), L_{U_{A}}\right)$. In other words, $\mathcal{Q}$ is a cone of $a^{\prime}(E): \operatorname{End}\left(E_{A}\right) \rightarrow L_{A}[1]$. Since the cup product of $a(E)$ with the Kodaira-Spencer class depends only on $a^{\prime}(E)$ (see the previous section) the obstruction module $O_{a}(M)$ can also be described as

$$
\operatorname{Coker}\left[\operatorname{Ext}_{U_{A}}^{1}(\mathcal{Q}, M) \rightarrow \operatorname{Ext}_{U_{A}}^{1}\left(L_{A}, M\right)\right]
$$

with appropriate $U_{A} \subset X_{A}$. Now apply étale localization for Ext $^{1}$ plus the identity $L_{B}=L_{A} \otimes_{A} B$ which holds for any étale extension $A \rightarrow B$, cf. Proposition III.3.1.1 and the exact sequence II.2.1.1.2 of [12].

Proposition 12 Let $\mathfrak{m} \subset A_{0}$ be a maximal ideal. Then

$$
D_{a_{0}}(M) \otimes_{A_{0}} \widehat{A}_{0} \simeq \lim _{\leftarrow} D_{a_{0}}\left(M / \mathfrak{m}^{n} M\right)
$$

and similarly for $\mathrm{Aut}_{a_{0}}\left(A_{0}+M\right)$.

Proof Suppose that $a_{0}$ is given by a pair $(E, U)$ as before. By Corollary 7 and discussion in Sect. 5.1 after a possible shrinking of $U$ we have

$$
D_{a_{0}}(M) \simeq H^{1}\left(U, E_{M}\right) ; \quad A u t_{a_{0}}\left(A_{0}+M\right) \simeq H^{0}\left(U, E_{M}\right) ;
$$

and similarly for the modules $M / \mathfrak{m}^{n} M$. Thus, we are required to show that formation of the two cohomology groups commutes with completion with respect to $\mathfrak{m}$. If $U=X_{A_{0}}$, then the projection $U \rightarrow \operatorname{Spec}\left(A_{0}\right)$ is proper and the assertion follows immediately by Theorem 4.1.5 in [7]. In general, denote by $j$ the open embedding of $U$ in $X_{A_{0}}$. The spectral sequence of $R \Gamma_{X} \circ R j_{*} \simeq R \Gamma_{U}$ gives in its lower terms $H^{0}\left(U, E_{M}\right) \simeq H^{0}\left(X_{A_{0}}, j_{*} E_{M}\right)$ and

$$
\begin{aligned}
0 & \rightarrow H^{1}\left(X_{A_{0}}, j_{*} E_{M}\right) \rightarrow H^{1}\left(U, E_{M}\right) \\
& \rightarrow H^{0}\left(X_{A_{0}}, R^{1} j_{*} E_{M}\right) \rightarrow H^{2}\left(X_{A_{0}}, j_{*} E_{M}\right) .
\end{aligned}
$$

By Corollary 7 , the sheaves $j_{*} E_{M}$ and $R^{1} j_{*} E_{M}$ on $X_{A_{0}}$ are coherent, and applying the same Theorem 4.1.5. in [7], we conclude that for these sheaves, cohomology commutes with completion. For $H^{0}\left(U, E_{M}\right)$ this immediately imples the result, while for $H^{1}\left(U, E_{M}\right)$ it follows after a standard diagram chase.

Proposition 13 Assume that the ring $A_{0}$ is reduced. Then there exists an open dense subset of points $p \in \operatorname{Spec}\left(A_{0}\right)$, so that

$$
D_{a_{0}}(M) \otimes_{A_{0}} k(p) \simeq D_{a_{0}}\left(M \otimes_{A_{0}} k(p)\right),
$$

and similarly for $\mathrm{Aut}_{a_{0}}\left(A_{0}+M\right)$ and $O_{a}(M)$. 
Proof We will write $X$ for $X_{A_{0}}$ to unload notation. Without loss of generality we can also assume that $\operatorname{Spec}\left(A_{0}\right)$ is irreducible, i.e., $A_{0}$ is a domain. Our proof is based on the following results:

- Exchange property. If $\pi: U \rightarrow S$ is a separated quasi-compact morphism and $\mathcal{F}$ is a coherent sheaf on $U$ which is flat over $S$ and such that $R^{i} \pi_{*} \mathcal{F}$ is flat over $S$ for any $i \geq 0$. Then $R^{i} \pi_{*}\left(\mathcal{F} \otimes_{\mathcal{O}_{X}} \pi^{*} \mathcal{M}\right) \simeq R^{i} \pi_{*}(\mathcal{F}) \otimes_{\mathcal{O}_{Y}} \mathcal{M}$. See Corollary 6.9.9 in [8].

- Generic freeness. If $A_{0}$ is a noetherian domain, $B$ a finitely generated noetherian $A_{0}$-algebra and $N$ a finitely generated $B$-module, then there exists a non-zero element $t \in A_{0}$ such that the localization $N_{t}$ is free over $\left(A_{0}\right)_{t}$. See Theorem 14.4 in [6].

Step 1. First we deal with Aut and $D$. The spaces $\operatorname{Aut}_{a_{0}}\left(A_{0}+M\right)$ and $D_{a_{0}}(M)$ were identified earlier with groups $H^{0}\left(U, \operatorname{End}(E)_{M}\right)$ and $H^{1}\left(U, \operatorname{End}(E)_{M}\right)$ where $U \subset X$ is some open subset in $\Phi\left(A_{0}\right)$ on which $\operatorname{End}(E)_{M}$ satisfies the relative $S_{3}$ condition (see the proof of Lemma 6). Thus, to prove the assertion for Aut $a_{0}(A+M)$ and $D_{a_{0}}(M)$ it suffices to show that for a vector bundle $P$ on $U$ after a single localization of $A_{0}$ we can achieve

$$
H^{i}\left(U, P_{M}\right) \simeq H^{i}(U, P) \otimes_{A_{0}} M ; \quad i=0,1
$$

for any finitely generated module $M$.

First, we reduce to the case of affine $X$. For the open embedding $j: U \rightarrow X$ the spectral sequence of $R \Gamma_{X} \circ R j_{*} \simeq R \Gamma_{U}$ gives $H^{0}\left(U, P_{M}\right) \simeq H^{0}\left(X, j_{*} P_{M}\right)$ and an exact sequence

$$
0 \rightarrow H^{1}\left(X, j_{*} P_{M}\right) \rightarrow H^{1}\left(U, P_{M}\right) \rightarrow H^{0}\left(X, R^{1} j_{*} P_{M}\right) \rightarrow H^{2}\left(X, j_{*} P_{M}\right) .
$$

Since $X$ is proper over $\operatorname{Spec}\left(A_{0}\right)$ all $H^{i}(X, \mathcal{F})$ are finitely generated over $A_{0}$ and since there are only finitely many non-zero cohomology groups, by Generic Freeness and the Exchange Property we can localize $A_{0}$ at powers of a nonzero element so that

$$
H^{i}\left(X, \mathcal{F}_{M}\right) \simeq H^{i}(X, \mathcal{F}) \otimes_{A_{0}} M, \quad i \geq 0
$$

for any finitely generated $A_{0}$-module $M$. Now denote the exact sequence (2) by $K^{\bullet}(M)$. Localizing $A_{0}$ further, we can assume that the cokernel of the last arrow in (2) is also free over $A_{0}$. Then, $K^{\bullet}\left(A_{0}\right)$ is a complex of projective $A_{0}$-modules and therefore $K^{\bullet}\left(A_{0}\right) \otimes_{A_{0}} M$ is exact. Comparing $K^{\bullet}\left(A_{0}\right) \otimes_{A_{0}} M$ with $K^{\bullet}(M)$ we reduce (1) to isomorphisms

$$
j_{*}(P)_{M} \simeq j_{*}\left(P_{M}\right), \quad R^{1} j_{*}(P)_{M} \simeq R^{1} j_{*}\left(P_{M}\right),
$$

which should hold after a further localization of $A_{0}$ (independent of $M$ ).

Step 2. By the previous step, assume that $X_{k}=\operatorname{Spec}(C)$ and $X=\operatorname{Spec}(B)$ with $B=C \otimes_{k} A_{0}$. We first show that one can localize $A_{0}$ and shrink $U$ to a smaller open subset $U^{\prime} \in \Phi\left(A_{0}\right)$ which can be covered by three affine open sets. Lemma 6 shows that such shrinking of $U$ will not change $j_{*}\left(P_{M}\right)$ and $R^{1} j_{*}\left(P_{M}\right)$. Indeed, let $I \subset B$ 
be an ideal with $V(I)=Z$. Choose a closed point $t \in \operatorname{Spec}\left(A_{0}\right)$. By assumption $X_{t}$ satisfies $S_{2}$ condition and $Z \cap X_{t}$ has codimension $\geq 3$ in $X_{t}$. Thus there exist $f, g \in I$ which form a regular sequence on $B^{\prime}:=B / \mathfrak{m}_{t} B$. Then the common zero set of $f, g$ has codimension 2 in $X_{t}$. Since $Z \cap X_{t}$ has codimension $\geq 3$ in $X_{t}$, by prime avoidance, cf. Lemma 3.3 in [6], one can find $h \in I$ such that the image of $h$ in $B^{\prime}$ does not belong to any codimension 2 prime associated to $B^{\prime} /(f, g) B^{\prime}$. Then, the ideal $(f, g, h) \in B$ is contained in $I$ and hence defines a closed subset $Z^{\prime} \supset Z$. By construction $Z^{\prime} \cap X_{t}$ has codimension $\geq 3$ in $X_{t}$ and by semicontinuity of dimension the same will hold for all fibers after a localization of $A_{0}$. The open complement $U^{\prime}$ of $Z^{\prime}$ is contained in $U$ and belongs to the family $\Phi\left(A_{0}\right)$. We replace $U, Z, I$ by $U^{\prime}, Z^{\prime},(f, g, h)$, hence assuming that $U$ is covered by the open subsets $U_{f}, U_{h}, U_{g}$ given by non-vanishing of $f, g, h$, respectively.

We want to apply the Exchange Property to $\pi: U \rightarrow \operatorname{Spec}\left(A_{0}\right)$. Since $U$ can be covered by three affine open $R^{i} \pi_{*}(P) \neq 0$ only for $i=0,1,2$, and these cohomology modules should become flat over $A_{0}$ after a localization of $A_{0}$. For $R^{0} \pi_{*}(P)$ and $R^{1} \pi_{*}(P)$ this follows from the Generic Freeness since the $B$-modules $N=H^{0}(U, P)$ and $H^{1}(U, P)$ are finitely generated. The module $H^{2}(U, P)$ is not finitely generated over $B$, but the distinguished triangle $R \Gamma_{Z} \rightarrow I d \rightarrow j_{*} j^{*} \rightarrow$ gives

$$
H^{2}(U, P) \simeq H_{I}^{3}(N) \simeq \lim _{\longrightarrow} N /\left(f^{n}, g^{n}, h^{n}\right) N
$$

where we used the last isomorphism before Appendix 4.1 in [6] and the fact that $I=(f, g, h)$. Since a filtered direct limit of projectives is flat, it suffices to show that $N_{n}=N /\left(f^{n}, g^{n}, h^{n}\right) N$ are projective over a dense open subset of $V \subset \operatorname{Spec}\left(A_{0}\right)$, for all $n \geq 1$. By a standard combinatorial argument $N_{n}$ admits a finite filtration with successive quotients of the type

$$
N_{p, q, r}=f^{p} g^{q} h^{r} N /\left(f^{p+1} g^{q} h^{r}, f^{p} g^{q+1} h^{r}, f^{p} g^{q} h^{r+1}\right) N
$$

and it suffices to ensure that these modules become free after a single localization of $A_{0}$. Indeed, each $N_{p, q, r}$ is isomorphic to a quotient of $N$ by a $B$-submodule $N_{p, q, r}^{\prime}$ and it is easy to see that $N_{p, q, r}^{\prime} \subset N_{p^{\prime}, q^{\prime}, r^{\prime}}^{\prime}$ if $p^{\prime} \geq p, q^{\prime} \geq q$ and $r^{\prime} \geq r$. The noetherian property of $N$ shows that $N^{\prime}=N_{p, q, r}^{\prime}$ for all but finitely many triples $(p, q, r)$, where $N^{\prime}$ is the union of all $N_{p, q, r}^{\prime}$. Hence, there are only finitely many isomorphism classes of $N_{p, q, r}$ and applying the Generic Freeness Lemma we can localize $A_{0}$ at powers of a single element and ensure that all $N_{p, q, r}$ are free over $A_{0}$. This finishes the proof of the isomorphisms $j_{*}(P)_{M} \simeq j_{*}\left(P_{M}\right)$ and $R^{1} j_{*}(P)_{M} \simeq R^{1} j_{*}\left(P_{M}\right)$ and therefore the assertion of the Proposition for Aut $a_{0}$ and $D_{a_{0}}$.

Step 3. To prove the assertion for $O_{a_{0}}(M)$ recall that by the proof of Proposition 11 we identified it with the cokernel of a morphism $\operatorname{Ext}_{U}^{1}(\mathcal{Q}, M) \rightarrow \operatorname{Ext}_{A_{0}}^{1}\left(L_{A_{0}}, M\right)$ which is natural in $M$. Therefore it suffices to prove isomorphisms

$$
\begin{aligned}
\operatorname{Ext}_{U}^{1}(\mathcal{Q}, M) & \simeq \operatorname{Ext}_{U}^{1}\left(\mathcal{Q}, A_{0}\right) \otimes_{A_{0}} M \\
\operatorname{Ext}_{A_{0}}^{1}\left(L_{A_{0}}, M\right) & \simeq \operatorname{Ext}^{1}\left(L_{A_{0}}, A_{0}\right) \otimes_{A_{0}} M
\end{aligned}
$$


after a localization of $A_{0}$. Both $\mathcal{Q}$ and $L_{A_{0}}$ are quasi-isomorphic to complexes of vector bundles in non-positive degrees, on $U$ and $\operatorname{Spec}\left(A_{0}\right)$, respectively. We will prove the assertion for $\mathcal{Q}$, the case of $L_{A}$ being entirely similar. By its definition $\mathcal{Q}$ is quasi-isomorphic to a complex of vector bundles $\cdots \rightarrow Q_{2} \rightarrow Q_{1} \rightarrow Q_{0}$ on $U$. Then a standard spectral sequence gives

$$
0 \rightarrow \operatorname{Ext}_{U}^{0}\left(Q_{1}, M\right) \rightarrow \operatorname{Ext}_{U}^{1}(\mathcal{Q}, M) \rightarrow \operatorname{Ext}_{U}^{1}\left(Q_{0}, M\right) \rightarrow \operatorname{Ext}_{U}^{0}\left(Q_{2}, M\right) \rightarrow \cdots
$$

It remains to apply $\operatorname{Ext}_{U}^{p}\left(Q_{q}, M\right) \simeq H^{p}\left(U, Q_{q}^{\vee} \otimes_{A_{0}} M\right)$ and the previous steps of the proof.

\section{Effectiveness}

Proposition 14 Let $\widehat{A}$ be a complete local algebra with residue field of finite type over $k$ and maximal ideal $\mathfrak{m}$; then the canonical functor

$$
F(\widehat{A}) \rightarrow \lim _{\longleftarrow} F\left(\widehat{A} / \mathfrak{m}^{n}\right)
$$

is an equivalence of categories.

Proof Let $\left\{\left(E_{n}, U_{n}\right) \in F\left(\widehat{A} / \mathfrak{m}^{n}\right)\right\}$ be a sequence representing an object on the righthand side. Shrinking $U_{n}$ as in Sect. 2 we can assume that each $E_{n}$ satisfies the relative $S_{3}$ condition on $U_{n}$. But then by the tangent-obstruction theory and stabilization of cohomology the set of isomorphism classes of extensions of $E_{n}$ to $F\left(A / \mathfrak{m}^{n+1}\right)$, i.e., the cohomology $H^{1}\left(\cdot, \operatorname{End}\left(E_{n}\right) \otimes \mathfrak{m}^{n} / \mathfrak{m}^{n+1}\right)$, will be the same over $U_{n}$ as over any open subset $W \subset U_{n} \cap U_{n+1}$ with closed complement in $\Phi\left(\widehat{A} / \mathfrak{m}^{n}\right)$. Therefore, we can assume that $E_{n+1}$ is defined also on $U_{n}$, and by induction all $E_{n}$ are defined on the same open subset $U^{\prime}$. Then by Theorem 6 in [B] there exists a bundle $E$ on an open subset $U \subset \operatorname{Spec}(\widehat{A})$ such that $(E, U)$ restricts to $\left(E_{n}, U^{\prime}\right)$ in each $F\left(A / \mathfrak{m}^{n}\right)$. On morphisms the assertion also follows from the main result of [4].

\section{Properties of the diagonal}

Lemma 15 Let $G$ be a coherent sheaf on $X_{k}$, A a noetherian k-algebra, and $H a$ coherent sheaf on $X_{A}$. Then there exists a finitely generated A-module $Q$, unique up to canonical isomorphisms, and a natural isomorphism of covariant functors (with argument $M$ )

$$
\operatorname{Hom}_{X_{A}}\left(H,\left(G \otimes_{k} A\right) \otimes_{A} M\right) \simeq \operatorname{Hom}_{A}(Q, M)
$$

from the category of A-modules to itself.

Proof First assume that $X_{k}$ is projective. Since $G \otimes_{k} A$ is flat over $A$ and $H$ is a cokernel of a morphism of locally free sheaves, the assertion is an immediate consequence of Corollary 7.7.8 in [8]. 
For proper $X_{k}$ we use the Chow lemma and the standard pattern of Sect. 5 in [7]. Fixing $H$, we will say that $G$ is representable if a module $Q$ as in the statement exists. Consider an exact sequence if coherent sheaves on $X_{k}$ :

$$
0 \rightarrow G_{1} \rightarrow G_{2} \rightarrow G_{3} \rightarrow 0
$$

We claim that if $G_{2}, G_{3}$ are representable, then $G_{1}$ is, and if $G_{1}, G_{3}$ are representable, then $G_{2}$ is. The first assertion is quite easy as the morphism $G_{2} \rightarrow G_{3}$ corresponds to the morphism $Q_{3} \rightarrow Q_{2}$ of representing $A$-modules, and we can set $Q_{1}=\operatorname{Coker}\left(Q_{3} \rightarrow Q_{2}\right)$. For the second assertion we first show that the functor $M \mapsto R(M)=\operatorname{Hom}_{X_{A}}\left(H,\left(G \otimes_{k} A\right) \otimes_{A} M\right)$ commutes with projective limits. In fact, choose an affine covering $X_{k}=\cup U_{i}$ and compute $R(M)$ as the kernel of the first arrow in the corresponding Cech complex

$$
\bigoplus_{i} \operatorname{Hom}_{\left(U_{i}\right)_{A}}\left(H,\left(G \otimes_{k} A\right) \otimes_{A} M\right) \rightarrow \bigoplus_{i \neq j} \operatorname{Hom}_{\left(U_{i} \cap U_{j}\right)_{A}}\left(H,\left(G \otimes_{k} A\right) \otimes_{A} M\right),
$$

where we omit the notation for restriction of sheaves to $U_{i}$ and $U_{i} \cap U_{j}$, respectively. Recall that projective limits are left exact and commute with $\operatorname{Hom}(H, \cdot)$. Hence by the universal property of projective limits it suffices to show that $\left(G \otimes_{k} A\right) \otimes_{A}(\cdot)$ commutes with projective limits, which is obvious since the first tensor factor is free over $A$. By Theorem 6 in [16], since $R(M)$ is left exact and commutes with projective limits, it is representable by an $A$-module $Q: R(M)=\operatorname{Hom}_{A}(Q, M)$ although in general $Q$ is not finitely generated. Now, if $G_{1}, G_{3}$ as above are represented by finitely generated $A$-modules $Q_{1}, Q_{3}$ and $G_{2}$ by an $A$-module $Q_{2}$, the exact sequence of sheaves induces an exact sequence of modules

$$
Q_{1} \rightarrow Q_{2} \rightarrow Q_{3} \rightarrow 0
$$

and since $Q_{1}, Q_{3}$ are finitely generated, the same holds for $Q_{2}$.

Now we prove the assertion for all proper $X_{k}$ by induction on the dimension of $\operatorname{Supp}(G)$ (as before, $A$ and $H$ are fixed). Choosing an appropriate closed subscheme in $X_{k}$ we can assume $\operatorname{Supp}(G)=X_{k}$. Then applying the Chow Lemma we find a projective subscheme $\widetilde{X}_{k}$ over $k$ and a projective morphism $h: \widetilde{X}_{k} \rightarrow X_{k}$ which is an isomorphism over a dense open subset of $X_{k}$. Let $h_{A}: \widetilde{X}_{A} \rightarrow X_{A}$ be the morphism obtained by base change $\operatorname{Spec}(A) \rightarrow \operatorname{Spec}(k)$. By adjunction

$$
\operatorname{Hom}_{\widetilde{X}_{A}}\left(h_{A}^{*} H,\left(h^{*}(G) \otimes_{k} A\right) \otimes M\right) \simeq \operatorname{Hom}_{X_{A}}\left(H,\left(h_{*} h^{*}(G) \otimes_{k} A\right) \otimes M\right),
$$

so the coherent sheaf $h_{*} h^{*}(G)$ is representable. Since the kernel and the cokernel of $\phi: G \rightarrow h_{*} h^{*}(G)$ are zero on a dense open subset of $X_{k}$, by induction they are representable. Hence, by the above argument $\operatorname{Im}(\phi)=\operatorname{Ker}\left(h_{*} h^{*}(G) \rightarrow \operatorname{Coker}(\phi)\right)$ is representable and from the exact sequence $0 \rightarrow \operatorname{Ker}(\phi) \rightarrow G \rightarrow \operatorname{Im}(\phi) \rightarrow 0$ the sheaf $G$ is also representable, as required. 
Corollary 16 Let $E$ be a vector bundle on $U=X_{A} \backslash Z$ with $Z \in \Phi(A)$ and $Y \rightarrow U$ a closed subscheme in the total space of $E$ over $U$. Then the functor $\operatorname{Sec}(Y / U)$ on $(A f f / A)$ which sends an A-algebra $B$ to the set of sections $U_{B}=U \times_{A} B \rightarrow Y_{B}=$ $Y \times{ }_{A} B$ is represented by an affine scheme of finite type over $A$.

Proof First we deal with $\operatorname{Sec}(E / U)$. By definition

$$
\operatorname{Sec}(E / U)(B)=\operatorname{Hom}_{\mathcal{O}_{U}-\operatorname{alg}}\left(\operatorname{Sym}^{\bullet}\left(E^{*}\right), \mathcal{O}_{U} \otimes_{A} B\right)=\operatorname{Hom}_{\mathcal{O}_{U}}\left(E^{*}, \mathcal{O}_{U} \otimes_{A} B\right)
$$

If $j: U \rightarrow X_{A}$ is the open embedding, then by adjunction $\operatorname{Hom}\left(j^{*}(\cdot), \cdot\right)=$ $\operatorname{Hom}\left(\cdot, j_{*}(\cdot)\right)$ and the $S_{2}$ condition we have

$$
\begin{aligned}
\operatorname{Hom}_{\mathcal{O}_{U}}\left(E^{*}, \mathcal{O}_{U} \otimes_{A} B\right) & =\operatorname{Hom}_{\mathcal{O}_{X_{A}}}\left(j_{*}(E), \mathcal{O}_{X_{A}} \otimes_{A} B\right) \\
& =\operatorname{Hom}_{A}(Q, B)=\operatorname{Hom}_{A-\operatorname{alg}}\left(\operatorname{Sym}_{A}^{\bullet}(Q), B\right)
\end{aligned}
$$

where the second equality holds for some finitely generated $A$-module $Q$ by the previous lemma. Thus, $\operatorname{Sec}(E / U)$ is represented by $\operatorname{Spec}\left(\operatorname{Sym}_{A}^{\bullet}(Q)\right)$.

For a closed subscheme $Y$ we can find a $\mathcal{O}_{U}$-coherent subsheaf $N$ of $\operatorname{Sym}_{\mathcal{O}_{U}}^{\bullet}\left(E^{*}\right)$ which generates the ideal subsheaf of $Y$ as $\operatorname{Sym}_{\mathcal{O}_{U}}^{\bullet}\left(E^{*}\right)$-module, e.g., by following the pattern of Proposition 9.6.5 in [7]. A section $s: U_{B} \rightarrow E_{B}$ induces a homomorphism of $\mathcal{O}_{U}$-algebras $\operatorname{Sym}_{\mathcal{O}_{U}}^{\bullet}\left(E^{*}\right) \rightarrow \mathcal{O}_{U} \otimes_{A} B$ and $s$ factors through $Y_{B}$ precisely when the restriction $\phi: N \rightarrow \mathcal{O}_{U} \otimes_{A} B$ vanishes. Fixing a coherent sheaf $N^{\prime}$ on $X_{A}$ which restricts to $N$ we get an isomorphism

$$
\operatorname{Hom}_{X_{A}}\left(N^{\prime}, \mathcal{O}_{X_{A}} \otimes B\right) \simeq \operatorname{Hom}_{U}\left(N, \mathcal{O}_{U} \otimes_{A} B\right)
$$

which follows by adjunction of $j^{*}, j_{*}$ and $j_{*}\left(\mathcal{O}_{U} \otimes_{A} B\right)=\mathcal{O}_{X_{A}} \otimes B$. By Lemma 15 there exists a finitely generated $A$-module $Q$ such that the above Hom groups can be identified with $\operatorname{Hom}_{A}(Q, B)$. Denote the corresponding homomorphism $Q \rightarrow B$ by the same letter $\phi$. Then for any $B$-algebra $B \rightarrow B^{\prime}$ the induced section $s_{B^{\prime}}$ corresponds to the composition of $\phi: Q \rightarrow B$ with $B \rightarrow B^{\prime}$. It follows that $s_{B^{\prime}}$ factors through $Y_{B^{\prime}}$ precisely when $\operatorname{Ker}\left(B \rightarrow B^{\prime}\right)$ contains the ideal generated by $\phi(Q) \subset B$. Therefore $\operatorname{Sec}(Y / U)$ is a closed subfunctor of $\operatorname{Sec}(E / U)$ and the assertion follows.

Proposition 17 The diagonal morphism of $F$ is representable, quasi-compact and separated.

Proof Although representability of the diagonal follows formally from the previous results, it is useful to establish it directly: if $S$ is an algebraic space, then a morphism $S \rightarrow F \times_{k} F$ corresponds to a pair of rank $r$ bundles $E_{1}, E_{2}$ which we may assume to be defined on a common open subset $X_{S} \backslash Z$. Then the fiber product with the diagonal is the functor of isomorphisms $\operatorname{Isom}\left(E_{1}, E_{2}\right)$. Although such isomorphisms correspond to sections with values in an open subset of the vector bundle $\operatorname{Hom}\left(E_{1}, E_{2}\right)$, the isomorphism condition is equivalent to the non-vanishing of the determinant, i.e., the induced section of $L=\operatorname{Hom}\left(\Lambda^{r} E_{1}, \Lambda^{r} E_{2}\right)$. Therefore, the subscheme of isomorphisms is isomorphic to the closed subscheme in the total space of $\operatorname{Hom}\left(E_{1}, E_{2}\right) \oplus L^{*}$, 
formed by all sections $(\phi, s)$ such that $\operatorname{det}(\phi) s=1$. Now Corollary 16 gives representability in the case when $S$ is affine, and uniqueness of the representing module $Q$ from Lemma 15 in the general case.

Quasi-compactness follows immediately from the fact that $\operatorname{Isom}\left(E_{1}, E_{2}\right)$ is affine over $S$.

By valuative criterion, cf. Theorem 7.8 in [14], separatedness of the diagonal morphism reduces to the following fact: if $R$ is a discrete valuation ring with residue field $K$ and $\left(E_{1}, U_{1}\right),\left(E_{2}, U_{2}\right)$ two objects in $F(R)$. Then a pair of isomorphisms between $E_{1}$ and $E_{2}$ that agree over $K$ must be equal over $R$. It suffices to show that $H^{0}\left(U_{1} \cap U_{2}, \operatorname{Hom}\left(E_{1}, E_{2}\right)\right)$ is torsion free. Denote $U=U_{1} \cap U_{2}, E=\operatorname{Hom}\left(E_{1}, E_{2}\right)$ and let $t \in R$ be a local parameter. Then we need to show that $t s=0$ for $s \in H^{0}(U, E)$ implies $s=0$. This question is local on $U$; hence we can assume that $E$ is a trivial bundle and $U=\operatorname{Spec}(B)$ for a flat $R$-algebra $B$. Then a short exact sequence $0 \rightarrow R \stackrel{t}{\longrightarrow} R \rightarrow K \rightarrow 0$ gives $0 \rightarrow B \stackrel{t}{\longrightarrow} B \rightarrow B \otimes_{R} K \rightarrow 0$, which proves the assertion.

Remark Observe that by Lemma 15 and the proof of Corollary 16, for any pair of bundles $E, F$ on $U=X_{A} \backslash Z$, the functor on $A$-modules, which sends $M$ to $\operatorname{Hom}_{U}\left(E, F \otimes_{A} M\right)$ is represented by a finitely generated $A$-module $Q$, unique up to canonical isomorphism: $\operatorname{Hom}_{U}\left(E, F \otimes_{A} M\right) \simeq \operatorname{Hom}_{A}(Q, M)$. Glueing such representing modules and applying generic freeness, one immediately obtains an analog of Proposition 2.2.3(i) in [13] which eliminates further possible pathologies of the diagonal morphism.

\section{Representability of principal bundles}

Proof of Theorem 1 To prove that $F_{G L(r)}$ is an algebraic stack, locally of finite type and separated over $k$, we just need to collect the results of the previous sections and compare with the conditions of Artin's representability criterion, cf. Theorem 5.3 in [1]. The "limit preserving" condition is proved in Sect. 3, Schlessinger's condition S1 in Sect. 4, while S2 is established in Sect. 5.1. Effectiveness (condition (2) of Artin's criterion) is given by Sect. 3, while part (3) of the criterion is proved in Sect. 5.2. Finally local quasi-separation (part (4) of Artin's criterion) is established in a stronger form in Sect. 7.

For a general reductive group $G$ over $k$ we use Theorem 3.3 in [11], and choose an exact finite dimensional representation $\rho: G \rightarrow G L(r)$ with $Y=G L(r) / G$ affine. Moreover, $Y$ is isomorphic to a closed $G L(r)$-orbit of a vector in a finite dimensional rational $G L(r)$-module $W$.

Then each principal $G$-bundle $P$ induces a principal $G L(r)$-bundle $E=P_{\rho}$. Conversely, for any principal $G L(r)$-bundle $E$ over a scheme $U$ its reduction of the structure group to $G$ may be viewed as a regular section $U \rightarrow Y_{E}=E \times_{G L(r)} Y$. Moreover, the scheme $Y_{E}$ which is affine and flat over $Y_{E}$ may be realized as a closed subscheme of a total space of a vector bundle $W_{E}$ on $U$, induced from $E$ via the homomorphism $G L(r) \rightarrow G L(W)$. 
This construction and Corollary 16 show that the morphism $F_{G} \rightarrow F_{G L(r)}$ is representable in the sense of Definition 3.9 in [14] and since $F_{G L(r)}$ is an algebraic stack, by Proposition 4.5(ii) of [14] $F(G)$ is also an algebraic stack.

Alternatively, we can re-prove the results of Sects. 3, 4 and 7 for $F_{G}$ by using the proved facts for vector bundles and reducing the structure group from $G L(r)$ to $G$. The analog of Proposition 12 in Sect. 6 is established by Theorem 13 of [4] using a similar strategy. Finally, by Sects. VI.2.4.2-VI.2.4.4 of [12] the arguments of Sect. 5 carry over to $G$ after a minor modification. Let $\Omega(G)$ be the space of $G$-invariant (from the right) differential forms on $G$, with the natural left $G$-action (in characteristic zero this is just the adjoint representation). For any $G$-bundle $P$ denote by $\operatorname{ad}(P)$ the vector bundle induced from $P$ via the action homomorphism $G \rightarrow G L(\Omega(G))$. Then all arguments of Sect. 5 carry over if $\operatorname{Ext}^{i}(\operatorname{End}(E), \cdot)$ are replaced by $\operatorname{Ext}^{i}(\operatorname{ad}(P), \cdot)$ and $H^{i}\left(\cdot, \operatorname{End}(E)_{M}\right)$ by $H^{i}\left(\cdot, \operatorname{ad}(P)_{M}^{\vee}\right)$, where $\operatorname{ad}(P)^{\vee}$ stands for the dual bundle. This finishes the proof of Theorem 1 .

Remark If we replace the "codimension 3" condition in the definition of $\Phi(T)$ (see Sect. 1), by "codimension 2", the stack $F_{G}$ will no longer be algebraic. The most obvious reason is that the tangent space $H_{A_{0}, \Phi}^{1}(\operatorname{End}(E))$ will no longer be finitely generated over $A_{0}$. On the other hand, the results of Sects. 3, 4 and 7 remain valid while the effectiveness of Sect. 6, which definitely fails by itself, may be repaired introducing an additional condition as in Corollary 8 of [4]. It is conjectured by V. Drinfeld that in this case $F_{G}$ is an inductive limit of algebraic stacks, locally of finite type over $k$. We plan to return to this topic, as well as to the related Uhlenbeck functor, in future work.

Open Access This article is distributed under the terms of the Creative Commons Attribution Noncommercial License which permits any noncommercial use, distribution, and reproduction in any medium, provided the original author(s) and source are credited.

\section{References}

1. Artin, M.: Versal deformations and algebraic stacks. Invent. Math. 27, 165-189 (1974)

2. Aoki, M.: HOM stacks. Manuscripta Math. 119(1), 37-56 (2006)

3. Aoki, M.: Erratum: "Hom stacks" Manuscripta Math. 121(1), 135 (2006)

4. Baranovsky, V.: Algebraization of bundles on non-proper schemes. Trans. Am. Math. Soc. 362, 427439 (2010)

5. Bruns, W., Herzog, J.: Cohen-Macaulay rings. In: Cambridge Studies in Advanced Mathematics, vol. 39. Cambridge University Press, Cambridge (1993)

6. Eisenbud, D.: Commutative algebra, with a view toward algebraic geometry. Graduate Texts in Mathematics, vol. 150. Springer, Berlin

7. Grothendieck, A.: EGA III 1 , "Étude cohomologique des faiseaux cohérents". Publ. Math. IHES (11), 5-167 (1961)

8. Grothendieck, A.: EGA III 2 , "Étude cohomologique des faiseaux cohérents". Publ. Math. IHES (17), 17-137 (1963)

9. Grothendieck, A.: EGA IV 2 , "Étude locale des schémas and des morphismes des schémas". Publ. Math. IHES (24), 5-231 (1965)

10. Grothendieck, A.: EGA $\mathrm{IV}_{3}$, "Étude locale des schémas and des morphismes des schémas". Publ. Math. IHES (28), 5-255 (1966)

11. Haboush, W.J.: Homogeneous vector bundles and reductive subgroups of reductive algebraic groups. Am. J. Math 100(6), 1123-1137 (1978) 
12. Illusie, L.: Complexe Cotangent et Déformations. Part I: LNM 239. Springer, Berlin (1971) (Part II: LNM 283, Springer (1972))

13. Lieblich, M.: Moduli of complexes on a proper morphism. J. Algebr. Geom. 15(1), 175-206 (2006)

14. Laumon, G., Morer-Bailly, L.: Champs algébriques. Springer, Berlin (2000)

15. Grothendieck, A., et al.: SGA 2, Cohomologie locale des faiseaux cohérents et théorèmes de Lefschetz locaux et globaux. Advanced Studies in Pure Mathematics, vol. 2, North-Holland, Amsterdam (1968)

16. Watts, C.E.: Intrinsic characterizations of some additive functors. Proc. Am. Math. Soc. 11, 5-8 (1960) 